\title{
Serum Phosphate Profile of Children With Severe Acute Malnutrition Treated With Locally Prepared Therapeutic Feeds: A Prospective Observational Study
}

\author{
Arunpandiyan Selvaraj ${ }^{a}$, Rajesh Sinha ${ }^{b}$, d, Preeti Singh ${ }^{a}$, \\ Anju Jain ${ }^{\mathrm{c}}$, Anju Seth ${ }^{\mathrm{a}}$, Praveen Kumara, d
}

\begin{abstract}
Background: Malnutrition in children $<5$ years is a public health concern due to its associated high morbidity, mortality and serious long-term consequences. The World Health Organization (WHO) recommends 10 steps for the management of severe acute malnutrition (SAM) children which also involves correction of electrolyte disturbances. Treatment with diets having inadequate amounts of minerals like phosphate can result in refeeding syndrome and other abnormalities. We conducted the study among children with medically complicated SAM to evaluate their serum phosphate levels after receiving locally prepared therapeutic feeds and its associations of other factors.
\end{abstract}

Methods: The study was conducted with 120 hospitalized SAM children aged 6 - 59 months with presence of other illnesses. All children were managed according to the WHO protocol and facility-based management of SAM guidelines of the government of India. Basic demographic details of the child were recorded. Weight of the child was recorded daily, while length/height and mid-upper arm circumference (MUAC) were recorded weekly. We evaluated changes in serum phosphate levels at three time points: on admission, during transition and at discharge. Binary logistic regression analysis was conducted to assess the association of hypophosphatemia with several other indicators.

Results: Mean serum phosphate was $4.38 \pm 1.07,4.48 \pm 1.16$ and 5.13 $\pm 1.10 \mathrm{mg} / \mathrm{dL}$ on admission, during transition and at discharge, re-

Manuscript submitted September 6, 2021, accepted September 26, 2021

Published online September 30, 2021

aDepartment of Peadiatrics, Lady Hardinge Medical College and Associated Kalawati Saran Children's Hospital, New Delhi 110001, India

bNational Centre of Excellence for SAM Management, Kalawati Saran Children's Hospital, New Delhi 110001, India

'Department of Biochemistry, Lady Hardinge Medical College and Associated Hospital, New Delhi 110001, India

${ }^{\mathrm{d} C}$ Corresponding Author: Rajesh Sinha, National Centre of Excellence for SAM Management, Kalawati Saran Children's Hospital, New Delhi 110001, India. Email: cmarajesh@gmail.com; Praveen Kumar, Lady Hardinge Medical College and Associated Kalawati Saran Children's Hospital, New Delhi 110001, India.Email: pkpaed@gmail.com

doi: https://doi.org/10.14740/ijcp458 spectively. Hypophosphatemia was present among 30 (25\%) children on admission, $28(23.3 \%)$ children during transition and $10(8.3 \%)$ children at discharge, which showed a decrease in the prevalence of hypophosphatemia from admission to transition and discharge using the current treatment. The finding also showed positive and significant changes in serum phosphate levels among children who were admitted without edema compared to those admitted with edema. Binary logistic regression estimates showed that older children, children with lower WAZ, hypokalemia, hyponatremia and anemia had higher odds of hypophosphatemia. Male children had lower odds of hypophosphatemia.

Conclusion: The nutritional therapy with locally prepared starter and catch-up diet was effective in normalizing hypophosphatemia in the majority of children treated. Serum potassium and serum sodium levels were significantly associated with serum phosphate, indicating the need for careful monitoring of serum phosphate in the presence of hypokalemia and hyponatremia among SAM children.

Keywords: Severe acute malnutrition; Facility-based management of SAM; Therapeutic diet; Edema; Hypophosphatemia; Hypokalemia; Hyponatremia

\section{Introduction}

Malnutrition in children less than 5 years is an important public health problem due to its associated high morbidity, mortality and serious long-term consequences $[1,2]$. Malnutrition exists in varying degrees of severity; children with severe acute malnutrition (SAM) are at maximum risk of adverse health outcomes and death.

Case fatality in SAM children is mostly attributed to systemic sepsis, hypoglycemia, hypothermia, dehydration, electrolyte imbalances, micronutrient deficiencies, infections and their consequences. Hence, World Health Organization (WHO) recommends 10 steps for the management of SAM children. Step 4 of the recommended 10 steps involves correction of electrolyte disturbances. Deficiencies of phosphorus, magnesium, potassium, calcium and other trace minerals are common in children with SAM [2]. Management in stabilization phase is focused upon correcting these factors by gradual refeeding with 
diet containing low calories, low protein and adequate amounts of minerals, vitamins and trace elements. Treatment with diets high in carbohydrate but with inadequate amount of phosphorus, magnesium, potassium and thiamine can result in refeeding syndrome which is characterized by hypophosphatemia, hypomagnesemia, hypokalemia and other abnormalities. On reviewing the literature, several studies reported varying prevalence of hypophosphatemia ranging from 17\% to 93\% [3-9].

Most of commercial premix (F-75) has added extrinsic phosphorus to meet United Nations procurement specifications of more than $60 \mathrm{mg} / 100 \mathrm{~mL}$. Locally prepared starter diet may have limited amounts of phosphorus during stabilization as compared to $60 \mathrm{mg} / \mathrm{kg} /$ day recommended by WHO and is likely to be less effective in normalizing phosphate levels which may lead to adverse outcomes like increasing mortality, inadequate weight gain, prolongation of hospital stay and other long-term morbidities [10-12]. With this background, we conducted the present study among children with medically complicated SAM in a large tertiary care hospital in New Delhi, India to evaluate serum phosphate levels in SAM children receiving locally prepared therapeutic feeds and correlation of other factors like age, sex, hypokalemia, hyponatremia, hypocalcemia, hypoparathyroidism, anemia, bilateral pitting edema, diarrhea, dehydration, vitamin $\mathrm{D}$ and other micronutrient deficiencies with incidence of hypophosphatemia.

\section{Materials and Methods}

The study was conducted between November 2018 to March 2020 with 120 children aged 6 - 59 months with SAM identified using weight-for-height $\mathrm{z}$-score $(\mathrm{WHZ})<-3$ and/or midupper arm circumference (MUAC) $<11.5 \mathrm{~cm}$ and/or bilateral pitting edema [13]. All children with SAM, except children with presence of known liver or renal disease or any childhood malignancy, were included in the study. Primary objective of the study was to evaluate changes in serum phosphate levels from the day of admission to transition (day 4 of admission), at discharge and correlation of serum phosphate levels with presence of hypokalemia, diarrhea, edema, 25(OH)VitD and parathyroid hormone $(\mathrm{PTH})$ abnormalities.

Basic demographic details (age, sex and religion) of the child were recorded. Weight of the child was recorded daily using electronic weighing machine (Seca 334). Length or height was recorded weekly using infantometer-cum-stadiometer. MUAC was measured weekly using a non-stretchable color coded tape to the nearest $1 \mathrm{~mm}$.

Other details such as dietary history, family history, socioeconomic history and immunization status of the child were also taken. Clinical examinations for presence of any new signs/symptoms were recorded for all enrolled children daily. All medical complications were managed according to the WHO protocol and facility-based management of severe acute malnutrition (FSAM) guidelines issued by the government of India [12]. Children discharged from the hospital after their medical complications were addressed, achieved average weight gain of $5 \mathrm{~g} / \mathrm{kg}$ body weight/day for three consecutive days, edema was treated and mothers/caregivers were counselled on essential nutrition practices.

Appetite test was conducted for all children before deciding on the initial diet. Children who passed the appetite were started with catch-up diet (F-100) and those who were failed were started with F-75 diet (all children in our study failed the appetite test and were started on F-75). These diets were prepared using locally available ingredients as per the protocol [11]. They received appropriate electrolytes, minerals and multivitamins as per the standard protocol such as potassium, magnesium, zinc, age-appropriate dose of vitamin A, vitamin D, vitamin K, folic acid and other multivitamins. Multivitamins, iron and folate supplementations were continued after discharge till the child recovered from malnutrition (WHZ $\geq$ -2). Additionally, the children and their family members were given individual and group therapy. Children were discharged from the hospital after meeting the discharge criteria as per the protocol [12].

\section{Sample collection}

Serum inorganic phosphate, serum potassium, serum sodium, and serum calcium (total and ionized) were measured on admission, on day 4 of admission and at discharge. Serum $25(\mathrm{OH})$ VitD and serum PTH were measured on admission. Sample for serum phosphate and other biochemical markers were collected in morning time in empty stomach or just before next feeding time in the plain vial. Serum PTH was collected in EDTA vial and centrifuged immediately in a cold centrifuge at $3,000 \mathrm{rpm}$ for $5 \mathrm{~min}$ and stored in a deep freeze at $-80^{\circ} \mathrm{C}$ for processing.

\section{Statistical analysis}

Data were entered in MS Excel and analyzed using IBM SPSS 20.0. Descriptive statistics like mean and standard deviation (SD) for continuous variables and percentages for categorical variables were done. $T$-test was used to compare two means and Chi-square test was used to compare proportions. Binary logistic regression analyses were performed to identify significant predictors of hypophosphatemia among children less than 5 years of age on admission. Independent variables taken in the analyses were age groups of children, gender, weight-forage z-score (WAZ), exclusive breastfeeding status, childhood illness (diarrhea with dehydration), micronutrient deficiency, anemia, hypokalemia (yes or no), hyponatremia (yes or no), hypocalcemia (yes or no), vitamin D deficiency and hypoparathyroidism (yes or no).

Dependent variable, i.e., serum phosphate profile, was categorial in nature with binary categories of 1 (hypophosphatemia) and 0 (normophosphatemia). All independent variables except WAZ were categorical in nature and binary categories were created with codes 1 and 0 for including them in the regression models. WAZ was taken as a continuous variable.

Children were also divided into two age groups (0 - 23 


\section{Total number of children under 5 years screened: 388}

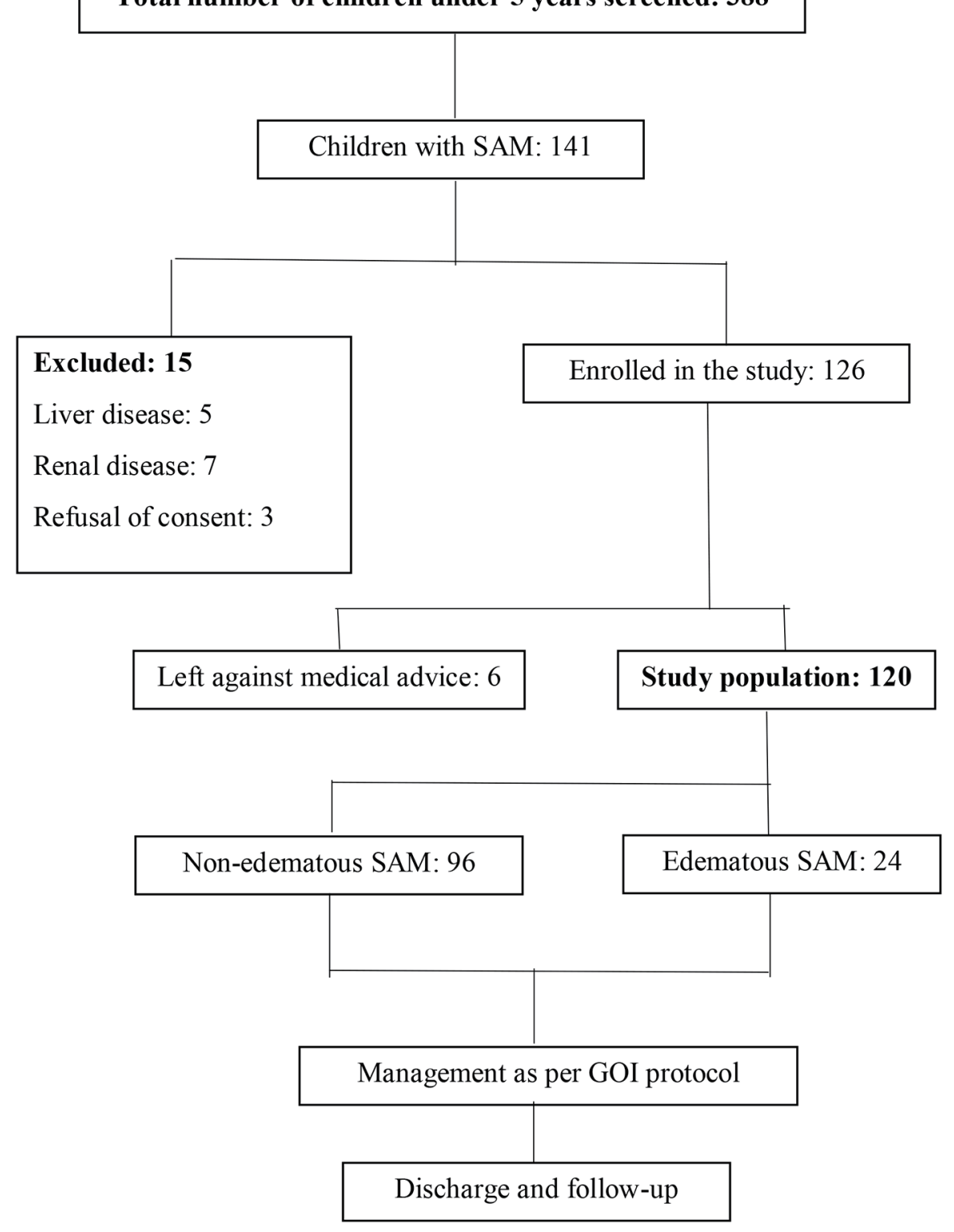

Figure 1. Schematic representation of enrolment of subjects. SAM: severe acute malnutrition.

months and 24 - 59 months) to understand whether serum phosphate profile was significantly different in early age group of children compared to the older children, keeping other variables constant.

\section{Ethical consideration}

Ethical clearance was taken from the institutional ethical committee and the study was conducted in compliance with all the applicable institutional ethical guidelines for the care, welfare and use of animals. The study was approved by Ethics Committee of Human Research of Lady Hardinge Medical College and Associated Hospitals, India vide letter number LHMC/
ECHR/2018/69 dated 26.10.2018.

\section{Results}

\section{Sample characteristics}

The study consisted of children between 6 and 59 months of age with SAM along with medical complications who were admitted in a tertiary care hospital in New Delhi, India. A total of 120 children were enrolled with mean age of $14.6 \pm 7.89$ months (Fig. 1). Out of these 120 children, 102 (85\%) were in the age group of 6 - 23 months and $65(54.2 \%)$ were female. 
Table 1. Socio-Demographic and Clinical Profile of Children Enrolled in the Study

\begin{tabular}{|c|c|}
\hline Variables & $\mathbf{N}(\%)$ \\
\hline \multicolumn{2}{|l|}{ Age group } \\
\hline $6-23$ months & $102(85.0)$ \\
\hline $24-59$ months & $18(15.0)$ \\
\hline \multicolumn{2}{|l|}{ Sex } \\
\hline Male & $55(45.8)$ \\
\hline Female & $65(54.2)$ \\
\hline \multicolumn{2}{|l|}{ Clinical profile on admission } \\
\hline Not growing well (parental perception) & $19(15.8)$ \\
\hline Diarrhea/vomiting & $56(46.7)$ \\
\hline Cough/difficulty in breathing & $28(23.4)$ \\
\hline Fever & $7(5.8)$ \\
\hline \multicolumn{2}{|l|}{ Other comorbid conditions } \\
\hline Anemia & $85(70.8)$ \\
\hline Rickets & $38(31.7)$ \\
\hline Celiac disease & $4(3.3)$ \\
\hline Hypothyroidism & $1(0.8)$ \\
\hline
\end{tabular}

Gastrointestinal complaints (diarrhea vomiting) were most common symptoms seen in $56(46.7 \%)$ subjects followed by respiratory complaints in $23.4 \%$ (cough, fast breathing, chest retraction and grunting) and not growing well in $15.8 \%$. Out of 56 subjects with loose stools, $45(80.3 \%)$ had dehydration of varying severity. Anemia was present in $85(70.8 \%)$ cases, while rickets was seen in $38(31.7 \%)$ subjects. Celiac disease was diagnosed in four $(3.3 \%)$ subjects and hypothyroidism in one $(0.8 \%)$ subject (Table 1$)$.

As shown in Table 2, mean weight was 6,146.17 $\pm 1,409.83$ $\mathrm{g}$ on admission which was increased to $6,395 \pm 1,413.51 \mathrm{~g}$ at discharge. The improvement in mean weight from admission to discharge was statistically significant $(\mathrm{P}<0.001)$. Mean WHZ, WAZ, height-for-age $\mathrm{z}$ score (HAZ) and MUAC were $-3.96 \pm 1.10,-4.18 \pm 1.16,-2.91 \pm 1.38$ and $108.02 \pm 9.43 \mathrm{~mm}$, respectively on admission. These values improved to $-3.48 \pm$
$1.00,-3.74 \pm 1.02,-2.82 \pm 1.30$ and $110.75 \pm 8.29 \mathrm{~mm}$, respectively at discharge. All these differences were statistically significant $(\mathrm{P}<0.001)$.

\section{Serum phosphate profile of children on admission, during transition and at discharge}

Mean serum phosphate was $4.38 \pm 1.07,4.48 \pm 1.16$ and 5.13 $\pm 1.10 \mathrm{mg} / \mathrm{dL}$ on admission, during transition and at discharge, respectively (Fig. 2). No child was found to have severe hypophosphatemia $(<1 \mathrm{mg} / \mathrm{dL})$ on anytime.

Hypophosphatemia was present in 30 (25\%) subjects on admission, $28(23.3 \%)$ subjects during transition and 10 $(8.3 \%)$ subjects at discharge. So, the prevalence of hypophosphatemia was decreased from admission to transition and discharge. On the other hand, prevalence of normophosphatemia was increased from admission to discharge with its prevalence of $71.7 \%, 71.7 \%$ and $80.8 \%$ on admission, during transition and at discharge, respectively (Table 3 ).

\section{Comparison of serum phosphate levels with other clinical characteristics present on admission}

Diarrhea was present in 56 (46.7\%) subjects on admission. Mean serum phosphate levels were $4.32,4.51$ and $5.20 \mathrm{mg} / \mathrm{dL}$ in children with diarrhea as compared to $4.43,4.45$ and $5.07 \mathrm{mg} / \mathrm{dL}$ in children without diarrhea on admission, during transition and at discharge, respectively. No statistically significant differences of mean serum phosphate were found between children with diarrhea and those without diarrhea on admission $(\mathrm{P}=0.569)$, during transition $(\mathrm{P}=0.772)$ or at discharge $(\mathrm{P}=0.569)$ (Table 4$)$.

Dehydration of varying severity was present in 45 (37.5\%) subjects on admission. Mean serum phosphate levels in subjects were $4.42,4.45$ and $5.2 \mathrm{mg} / \mathrm{dL}$ in children with dehydration as compared to $4.34,4.48$ and $5.09 \mathrm{mg} / \mathrm{dL}$ in children without dehydration on admission, during transition and at discharge, respectively. No statistically significant differences of mean serum phosphate were found between children with dehydration and those without dehydration on admission $(\mathrm{P}=0.693)$, during transition $(\mathrm{P}=0.891)$ or at discharge $(\mathrm{P}=0.578)($ Table 4$)$.

Table 2. Anthropometric Parameters/Indices on Admission and at Discharge

\begin{tabular}{llll}
\hline Variables & On admission, mean (SD) & At discharge, mean (SD) & $t$-statistics/Chi-square statistics, P-value \\
\hline Weight $(\mathrm{g})$ & $6,146.17(1,409.83)$ & $6,395.42(1,413.51)$ & $t$-statistics $=9.30, \mathrm{P}<0.001$ \\
WAZ & $-4.18(1.16)$ & $-3.74(1.02)$ & $t$-statistics $=10.69, \mathrm{P}<0.001$ \\
HAZ & $-2.91(1.38)$ & $-2.82(1.30)$ & $t$-statistics $=4.00, \mathrm{P}<0.001$ \\
WHZ & $-3.96(1.10)$ & $-3.48(1.00)$ & $t$-statistics $=10.92, \mathrm{P}<0.001$ \\
MUAC $(\mathrm{mm})$ & $108.02(9.43)$ & $110.75(8.29)$ & $t$-statistics $=7.63, \mathrm{P}<0.001$ \\
WHZ $<-3$ & $103(85.80)$ & $82(68.30)$ & Chi-square statistics $=10.35, \mathrm{P}=0.001$ \\
MUAC $<115 \mathrm{~mm}$ & $92(76.70)$ & $83(69.20)$ & Chi-square statistics $=1.70, \mathrm{P}=0.19$ \\
Bilateral pitting edema & $24(20.0)$ & $0(0.00)$ & Chi-square statistics $=26.56, \mathrm{P}<0.001$ \\
\hline
\end{tabular}

WAZ: weight-for-age z-score; HAZ: height-for-age z-score; WHZ: weight-for-height z-score; MUAC: mid-upper arm circumference; SD: standard deviation. 


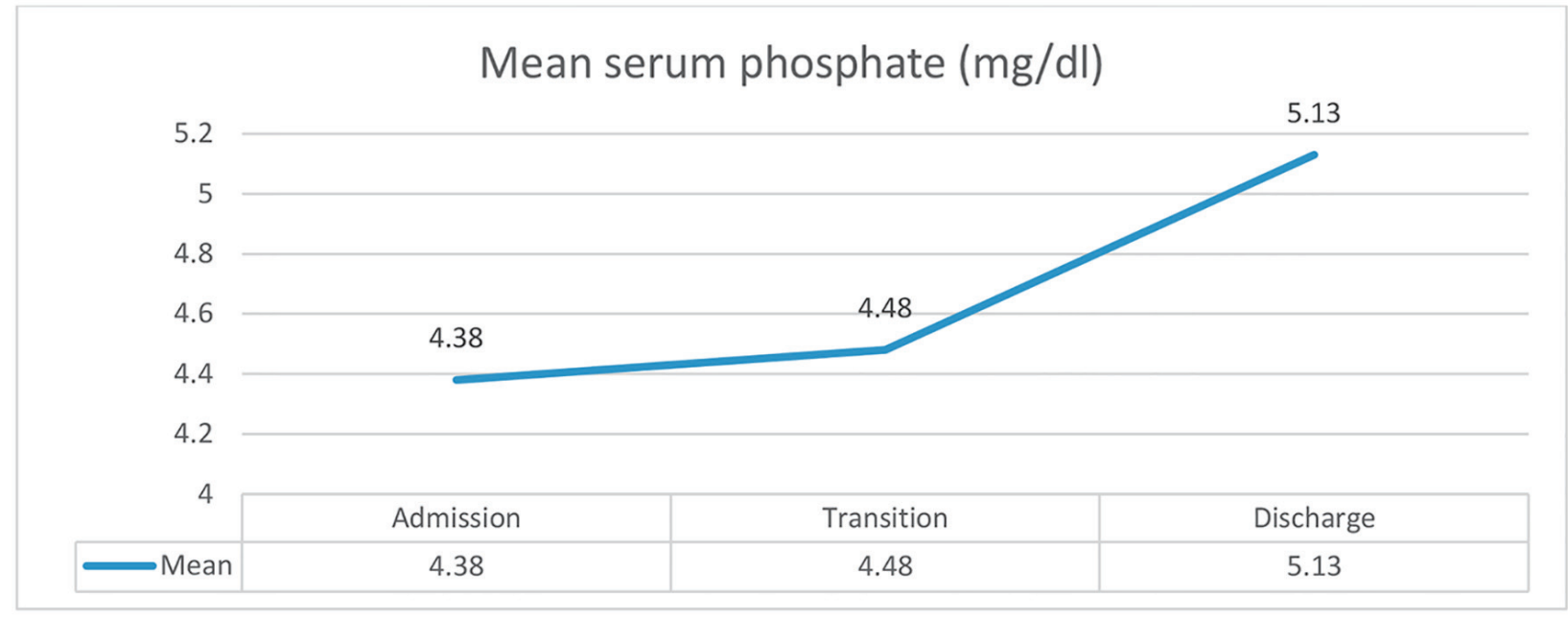

Figure 2. Serum phosphate profile of children on admission, during transition and at discharge.

Table 3. Changes in Serum Phosphate Profile From Admission to Discharge

\begin{tabular}{llll}
\hline Phosphate profile & Admission, N (\%) & Transition, N (\%) & Discharge, N (\%) \\
\hline Normophosphatemia & $86(71.7)$ & $86(71.7)$ & $97(80.8)$ \\
Hypophosphatemia & $30(25.0)$ & $28(23.3)$ & $10(8.3)$ \\
Hyperphosphatemia & $4(3.3)$ & $6(5.0)$ & $13(10.8)$ \\
Total & $120(100.0)$ & $120(100.0)$ & $120(100.0)$ \\
\hline
\end{tabular}

Table 4. Comparison of Serum Phosphate Levels on Admission, Transition and Discharge in Subjects With Different Clinical Characteristics Present on Admission

\begin{tabular}{|c|c|c|c|c|}
\hline Mean serum phosphate & Clinical characteristics on admission & Status & Mean (SD), mg/dL & P value* \\
\hline \multirow[t]{2}{*}{ Admission } & Diarrhea & Yes & $4.32(1.07)$ & 0.569 \\
\hline & & No & $4.43(1.06)$ & \\
\hline Transition & & No & $4.44(0.98)$ & \\
\hline Discharge & & Yes & $5.19(1.10)$ & 0.569 \\
\hline Admission & & No & $4.34(1.08)$ & \\
\hline \multirow[t]{2}{*}{ Transition } & & Yes & $4.45(1.30)$ & 0.891 \\
\hline & & No & $4.48(1.08)$ & \\
\hline Discharge & & Yes & $5.2(1.22)$ & 0.578 \\
\hline \multirow[t]{2}{*}{ Transition } & & Yes & $4.21(1.16)$ & 0.211 \\
\hline & & No & $4.54(1.16)$ & \\
\hline \multirow[t]{2}{*}{ Discharge } & & Yes & $4.79(1.13)$ & 0.089 \\
\hline & & No & $5.22(1.08)$ & \\
\hline
\end{tabular}

*Unpaired t-test. SD: standard deviation. 
Table 5. Comparison of Serum Phosphate Profile on Admission, Transition and Discharge Between Children With Edema and Without Edema on Admission

\begin{tabular}{llllll}
\hline \multirow{2}{*}{ Admission } & Edema on admission & Hypophosphatemia & Normophosphatemia & Hyperphosphatemia & P value* \\
& Yes & $8(33.33 \%)$ & $16(66.67 \%)$ & $0(0 \%)$ & 0.379 \\
\multirow{2}{*}{ Transition } & No & Yes & $22(22.90 \%)$ & $70(72.70 \%)$ & $4(4.2 \%)$ \\
& No & $11(45.82 \%)$ & $12(50 \%)$ & $5(4.2 \%)$ & 0.014 \\
\multirow{2}{*}{ Discharge } & Yes & $17(17.7 \%)$ & $74(77 \%)$ & $3(13 \%)$ & $13(13.4 \%)$ \\
& No & $5(20.8 \%)$ & $16(66.67 \%)$ & 0.040 \\
\hline
\end{tabular}

${ }^{*}$ Chi-square test.

Similarly, mean serum phosphate was $4.31,4.21$ and 4.79 $\mathrm{mg} / \mathrm{dL}$ in subjects with edema, while it was 4.39, 4.54 and 5.22 $\mathrm{mg} / \mathrm{dL}$ in cases of children without edema on admission, during transition and at discharge, respectively. The mean difference of levels of serum phosphate was statistically non-significant between children with edema and those without edema on admission and during transition; however, it was partially significant at discharge $(\mathrm{P}=0.09)$ (Table 4).

On admission, majority of children with $(66.7 \%)$ or without $(72.7 \%)$ edema had serum phosphate profile within the normal range and the difference in prevalence of different serum phosphate profiles between children with edema and those without edema was found to be non-significant $(\mathrm{P}=0.38)$. However, during the transition phase, around $45.8 \%$ of children with edema had hypophosphatemia, while $77 \%$ children without edema had serum phosphate profile within the normal range. Difference in prevalence of different serum phosphate profiles between children with edema and those without edema was found to be significant during the transition phase $(\mathrm{P}=$ $0.01)$. At discharge, $84.4 \%$ children without edema had serum phosphate profile within the normal range, while it was only among $66.67 \%$ children with edema. Difference in prevalence of different serum phosphate profiles between children with edema and those without edema was found to be significant at discharge $(\mathrm{P}=0.04)$. The above findings show that positive and significant changes in serum phosphate profile were observed among children without edema compared to children with edema from admission to discharge (Table 5).

\section{Predictors of hypophosphatemia}

Binary logistic regression estimates showed that older children had higher odds of hypophosphatemia. Male children had lower odds of hypophosphatemia. Children with lower WAZ had higher odds of hypophosphatemia. Children with hypokalemia and hyponatremia had higher odds of hypophosphatemia. Similarly, anemic children had higher odds of hypophosphatemia. No significant associations of hypophosphatemia were found with hypocalcemia, hypoparathyroidism, vitamin D deficiency, deficiency in other micronutrient, bilateral pitting edema and diarrhea with dehydration (Table 6).

Table 6. Predictors of Hypophosphatemia Among Children on Admission

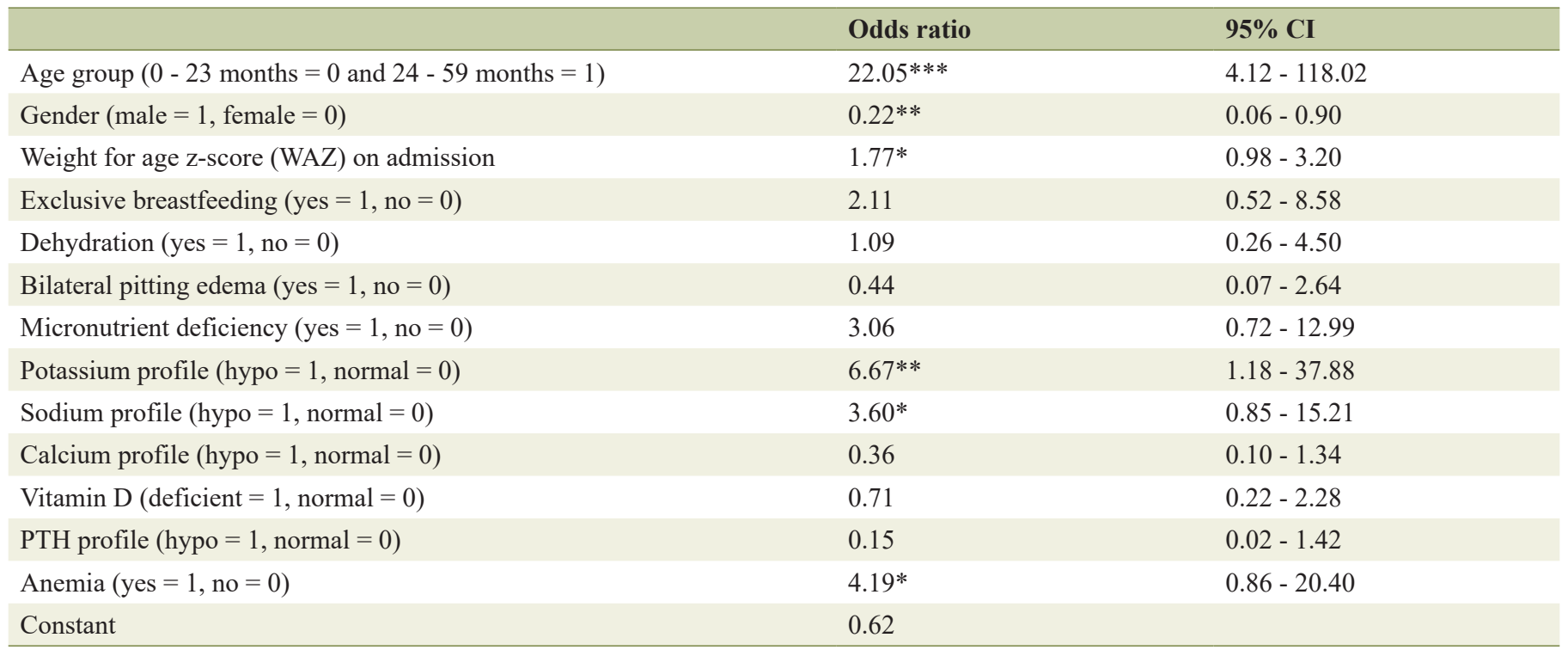

${ }^{* * *} \mathrm{P}<0.01,{ }^{* *} \mathrm{P}<0.05,{ }^{*} \mathrm{P}<0.10$. WAZ: weight-for-age $\mathrm{Z}$-score; $\mathrm{PTH}$ : parathyroid hormone; Cl: confidence interval. 


\section{Discussion}

SAM is a public health problem of immense importance worldwide especially in developing countries like India. The children with SAM are at higher risk of deaths when they develop medical complications. Recognizing this high risk, WHO laid out guidelines for management of complicated SAM cases at facility level. In 2011, the MoHFW, GoI also published technical and operational guidelines for the management of medically complicated SAM cases at facility level. Nutritional rehabilitation along with medical care to SAM children with medical complications is required to prevent mortality and long-term complications. Since starter diet has low phosphorus, there is a risk of hypophosphatemia particularly in stabilization phase. Moreover, in India, locally prepared starter and catch-up diets are being used in place of commercial F-75 and F-100. Very few studies have been conducted regarding the incidence of hypophosphatemia and other electrolyte abnormalities during rehabilitation of SAM children using locally prepared therapeutic diet in health-care settings.

In our study, mean serum phosphate was $4.38,4.48$ and $5.13 \mathrm{mg} / \mathrm{dL}$ on admission, during transition and at discharge, respectively, showing increasing trend throughout the course of stay. Other studies reported mean serum phosphate levels lesser than $4 \mathrm{mg} / \mathrm{dL}$ on admission and transition. Also, the level usually fell from admission for first few days, as starter diet contains limited phosphate before it rises once catch-up diet with more phosphate is introduced [3, 7]. Mean serum phosphate at discharge was comparable to studies done by Namusoke et al [5], but overall mean increase of $0.75 \mathrm{mg} / \mathrm{dL}$ in our study was less than $2.07 \mathrm{mg} / \mathrm{dL}$ reported by Namusoke et al.

Prevalence of hypophosphatemia in our study was $25 \%$, $23.3 \%$ and $8.3 \%$ on admission, during transition and at discharge, respectively. The prevalence on admission was comparable with studies by Namusoke et al $(37 \%)$ and Dakshayani et al $(17 \%)[5,8]$, while Kimutai et al $(86 \%)$, Hother et al $(93 \%)$ and Chanchal et al $(60 \%)$ reported very high prevalence $[3,4$, 7]. Higher prevalence in the study by Kimutai et al could be explained by difference in study population as they enrolled only children with edematous malnutrition.

In our study, hypophosphatemia was still persisting in $8.3 \%$ subjects at discharge. This is in agreement with studies done by Namusoke et al (6\%) and Dakshayani et al $(17 \%)$. Thus, our study suggests local starter and catch-up diets prepared as per WHO recommendation (without added phosphate) are efficacious in correcting hypophosphatemia in majority of cases. Mean serum phosphate levels in our study were relatively higher as compared to other studies which may be explained by higher intake dairy based diet that contains higher phosphate content. The above reason also explains efficacy of locally prepared diets (without phosphorus supplementation) in correcting hypophosphatemia.

There was no significant difference in phosphate levels of children with or without diarrhea and dehydration. This was in accordance with studies done by Kimutai et al [3], Hother et al [4] and Okinyi et al [9]. However, Namusoke et al [5] reported a significant difference in hypophosphatemia prevalence at transition in children with or without diarrhea. This difference may be due to multiple factors, as all who had diarrhea re- ceived rice porridge, which contains lesser phosphate (even lesser than F-75) and higher carbohydrate composition causing hypophosphatemia while remaining children who received premixed F-75 as per the WHO recommendation did not have hypophosphatemia [5].

Prevalence of edema was $20 \%$ in our study which was higher than an Indian study done by Chanchal et al [7] which reported $12.3 \%$. Serum phosphate varied significantly between subjects with edema and without edema, with mean difference of $0.08,0.33$ and $0.43 \mathrm{mg} / \mathrm{dL}$ on admission, during transition and at discharge, respectively. Prevalence of hypophosphatemia on admission, during transition and at discharge was $33.3 \%$, $45.8 \%$ and $20.8 \%$ in subjects with edema and $22.9 \%, 17.7 \%$ and $5.2 \%$ in subjects without edema. Statistically significant difference was found between the two groups on transition ( $\mathrm{P}$ $=0.014)$ and discharge $(\mathrm{P}=0.04)$. This finding is in accordance with most of studies in which prevalence of edema was high in the study subjects [6-8].

Significant associations were found between hypophosphatemia with hypokalemia and hyponatremia on admission. Yoshimatsu et al reported significant association of hypokalemia and hypophosphatemia in SAM children [14]. Also, concomitant occurrence of hypophosphatemia and hypokalemia during nutritional rehabilitation of malnourished children was well known. To our knowledge, no other study reported any significant association of serum phosphate with serum sodium levels in nutritional rehabilitation of SAM children. Similarly, hypophosphatemia was significantly associated with anemia in our study but Chanchal et al [7] reported no association between the two.

As vitamin D and PTH have very close relationship with serum phosphate and in turn bone metabolism under normal condition, its association with serum phosphate in SAM children becomes important. Vitamin D deficiency was not associated with hypophosphatemia. High prevalence of vitamin D deficiency may precipitate and increase overall prevalence of hypophosphatemia and its associated complications. Similarly, hypophosphatemia on admission was not significantly associated with serum PTH levels. To our knowledge, no other studies have compared the prevalence of hypophosphatemia with vitamin D deficiency and PTH status in SAM children.

\section{Strength and limitations of study}

As this study was conducted in a large government run hospital in the country, the findings represent a wide range of population and can therefore be generalized for all SAM children with medical complications. However, a multicenter study with large sample size needs to be conducted in future to evaluate the association of hypophosphatemia among medically complicated SAM children with other factors. There are a few limitations of the study. Serum magnesium was not measured as it was not feasible in our hospital at the time of study period. We also faced difficulties in obtaining fasting samples in very small children because of frequent feeding timings. This may have led to measure falsely increased phosphate levels in serum. Also, we did not conduct post-discharge follow-ups of these children to assess further weight gain and other parameters. We did not conduct study with sick children requiring pediatric intensive care 
unit (PICU) admission, ventilators, and inotropes as they were frequently off feeds/on intravenous fluid (IVF)/inotropes.

\section{Conclusion}

In our study, hypophosphatemia was present in $25 \%$ children on admission. The nutritional therapy with locally prepared starter and catch-up diet was effective in normalizing hypophosphatemia in two-thirds of children as mild hypophosphatemia was still present in $8.3 \%$ of children at discharge. This may hamper the growth after discharge, necessitating the importance of frequent clinical follow-up and ongoing nutritional supplementation in community. Serum potassium and serum sodium levels were significantly associated with serum inorganic phosphate, indicating the need of careful monitoring of serum phosphate in the presence of hypokalemia and hyponatremia.

\section{Acknowledgments}

The authors acknowledge the time given by the caregivers of all the study subjects for participating in the study.

\section{Financial Disclosure}

No funding support was received for the study.

\section{Conflict of Interest}

The authors declare no conflict of interest.

\section{Informed Consent}

Written informed consent was obtained from the caregivers of the study subjects.

\section{Author Contributions}

ApS, PS, AJ, AS and PK conceived the study. RS and ApS analyzed the data. RS and ApS wrote the manuscript and collated subsequent inputs. All authors reviewed and approved the manuscript.

\section{Data Availability}

The authors declare that data supporting the findings of this study are available within the article.

\section{Reference}

1. Rice AL, Sacco L, Hyder A, Black RE. Malnutrition as an underlying cause of childhood deaths associated with infectious diseases in developing countries. Bull World Health Organ. 2000;78(10):1207-1221.

2. Pelletier DL, Frongillo EA, Jr., Schroeder DG, Habicht JP. A methodology for estimating the contribution of malnutrition to child mortality in developing countries. J Nutr. 1994;124(10 Suppl):2106S-2122S.

3. Kimutai D, Maleche-Obimbo E, Kamenwa R, Murila F. Hypo-phosphataemia in children under five years with kwashiorkor and marasmic kwashiorkor. East Afr Med J. 2009;86(7):330-336.

4. Hother AL, Girma T, Rytter MJ, Abdissa A, Ritz C, Molgaard C, Michaelsen KF, et al. Serum phosphate and magnesium in children recovering from severe acute undernutrition in Ethiopia: an observational study. BMC Pediatr. 2016;16(1):178.

5. Namusoke H, Hother AL, Rytter MJ, Kaestel P, Babirekere-Iriso E, Fabiansen C, Girma T, et al. Changes in plasma phosphate during in-patient treatment of children with severe acute malnutrition: an observational study in Uganda. Am J Clin Nutr. 2016;103(2):551-558.

6. Rytter MJ, Babirekere-Iriso E, Namusoke H, Christensen VB, Michaelsen KF, Ritz C, Mortensen CG, et al. Risk factors for death in children during inpatient treatment of severe acute malnutrition: a prospective cohort study. Am J Clin Nutr. 2017;105(2):494-502.

7. Chanchal R, Gupta S, Kanta C, Singh K, Koonwar S. Hypophosphataemia in severe acute malnutrition: a prospective observational study. Br J Nutr. 2019;121(3):306311.

8. Dakshayani B, Divyashree P, Sabapathi S, Kariyappa M. Changes in serum phosphorous level during inpatient treatment of children with severe acute malnutrition. International Journal of Contemporary Pediatrics. 2019;6(3):1080.

9. Okinyi LK. The prevalence of refeeding syndrome among children with severe acute malnutrition: an observational study in Kenyatta National Hospital, Kenya. Med Clin Res. 2018;3(2):1-4.

10. WHO. Management of severe malnutrition: a manual for physicians and other senior health workers. Geneva, World Health Organization; 1999.

11. Bhatnagar S, Lodha R, Choudhury P, Sachdev HP, Shah $\mathrm{N}$, Narayan S, Wadhwa N, et al. IAP guidelines 2006 on hospital based management of severely malnourished children (adapted from the WHO Guidelines). Indian Pediatr. 2007;44(6):443-461.

12. Operational guidelines on facility-based management of children with severe acute malnutrition. Ministry of Health and Family Welfare, Government of India, New Delhi. 2011.

13. World Health Organization. Adapted from WHO growth standards and identification of severe acute malnutrition in infants and children. A joint statement of WHO and UNICEF. 2009. Geneva; 2009. p. 2.

14. Yoshimatsu S, Hossain MI, Islam MM, Chisti MJ, Okada M, Kamoda T, Fukushima T, et al. Hypophosphatemia among severely malnourished children with sepsis in Bangladesh. Pediatr Int. 2013;55(1):79-84. 